\title{
SOBREPESO: UMA NOVA REALIDADE NO ESTADO NUTRICIONAL DE PRÉ-ESCOLARES DE NATAL, RN
}

O Século XX caracterizou-se pelo importante progresso científico e tecnológico, que propiciou grandes conquistas na saúde dos indivíduos e na busca pela melhor qualidade de vida, principalmente para crianças e adolescentes. Testemunhamos a significativa diminuição nos índices de morbimortalidade na infância, por duas causas importantes: doenças infecto-contagiosas, graças ao advento das vacinas e da terapêutica antimicrobiana, e doença diarréica aguda, com o uso da terapia de reidratação oral.

Por outro lado, porém, os processos de industrialização, urbanização e as decorrentes mudanças na estrutura social, como por exemplo, o ingresso da mulher no mercado de trabalho e a crescente violência urbana, acarretaram em profundas alterações nos hábitos de vida da população. Observamos mudanças estruturais nos hábitos alimentares de crianças e adolescentes, com maior ingestão de gorduras e açúcares e menor consumo de alimentos ricos em fibras. Observamos também o aumento do sedentarismo nesta faixa etária, propiciado pelo lazer inativo com televisão, computador e vídeo game. Assim, crianças e adolescentes tornaram-se cada vez mais vulneráveis ao excesso de peso, como ocorre na epidemia global de obesidade adulta, inclusive com a presença de resistência à insulina, diabetes mellitus tipo II e aterosclerose precoce, compondo o quadro de síndrome metabólica ${ }^{7,8}$.

A prevalência de obesidade e sobrepeso em crianças e adolescentes, de 2 a 17 anos, que nas duas últimas décadas passou a mostrar um perfil epidêmico, com tendência à pandemia, é de $10,1 \%$ e $8,5 \%$, respectivamente. Nos adolescentes, a prevalência é de $3 \%$ 2,8,9.

A prevalência de sedentarismo em crianças e adolescentes varia bastante, dependendo dos critérios utilizados². Sabe-se que a atividade física de forma regular tem múltiplas vantagens: ajuda no desenvolvimento e na manutenção das articulações, músculos e ossos; auxilia no controle do peso, reduzindo a gordura e aumentando a massa muscular; previne ou retarda o desenvolvimento de hipertensão arterial sistêmica; ajuda a reduzir os níveis de pressão arterial em adolescentes já hipertensos; diminui a incidência da maioria das doenças crônico-degenerativas, como as doenças cardiovasculares e contribui significativamente para reduzir os sentimentos de depressão e ansiedade ${ }^{7,8}$.

Observa-se atualmente que a atividade física tem diminuído entre crianças e adolescentes, e o momento de lazer em frente à televisão, ao vídeo game e ao computador passa a fazer parte do seu estilo de vida durante muitas horas por dia. A criança de hoje gasta em média 600 quilocalorias (Kcal) diárias a menos que 50 anos atrás. As atividades infantis passaram a ser dentro de casa, onde a criança assiste hoje, em média, a 27 horas de televisão por semana, o que corresponde à sua principal atividade. Este fenômeno reflete a falta de áreas de lazer com segurança nas grandes metrópoles, restringindo, com isso, o engajamento das crianças nas atividades de recreação ou esportivas ${ }^{7,8}$.
A atividade física regular vem sendo recentemente listada como um dos principais indicadores de saúde. Segundo o Center for Disease Control (CDC), todos os indivíduos, a partir dos dois anos de idade, que não desenvolvem 30 minutos de atividade física, de moderada a intensa, durante a maioria (cinco dias) ou, preferencialmente, todos os dias da semana, são considerados sedentários. A avaliação da atividade física deve ser examinada em relação ao tempo e ao nível do exercício, bem como o tempo gasto com atividades físicas junto com à família. Por outro lado, também é conveniente verificar o tempo despendido com jogos eletrônicos, televisão e computador. O tempo de inatividade recreacional deve ser limitado a duas horas por dia, no máximo!.

Assim, os hábitos de vida da criança e do adolescente de hoje induzem à inatividade física, ao sedentarismo, à alimentação inadequada e, conseqüentemente, ao aumento nos índices de obesidade, como bem aponta o trabalho de Barreto et. al. Estudos como este são fundamentais para que se possa ter uma noção dos índices de obesidade e sobrepeso em cada região e para que medidas possam ser tomadas no sentido de minimizar os efeitos a longo prazo da obesidade e de suas comorbidades.

As conseqüências da obesidade e da inatividade física na saúde do indivíduo tornam-se aparentes na vida adulta. É fundamental, porém, ter a visão de que refletem, na verdade, hábitos adquiridos na infância.

Sabe-se hoje que importantes causas de óbito na vida adulta são as doenças crônico-degenerativas, principalmente as doenças cardiovasculares. É, portanto, imperioso entender que estas doenças têm suas raízes na infância. Estimular hábitos de vida saudáveis, como alimentação adequada e exercícios físicos, é tarefa fundamental do pediatra que faz puericultura para a criança que pretende viver 100 anos com qualidade de vida.

Pais e escolas têm um papel de suma importância na orientação e na conduta a serem transmitidas para as crianças e os adolescentes. A correria do dia-a-dia, que facilita o consumo de lanches rápidos, e a escola, que na maioria das vezes oferece alimentos gordurosos e de baixo valor nutritivo, além da atividade física pouco expressiva no ambiente escolar, devem ser redirecionados a uma nova forma de pensar e de conduzir ações que visem proporcionar um estilo de vida mais saudável.

aconselhamento deve incluir estratégias comprometidas com dieta saudável e atividade física. A dieta pobre em gorduras saturadas e colesterol, que inclua cinco ou mais refeições diárias compostas por vegetais e frutas, de seis a II porções de grãos integrais e outros alimentos com carboidratos complexos, é a dieta de escolha para a prevenção da obesidade e co-morbidades ${ }^{4,8}$.

Pode ser útil para pais, educadores e crianças visualizarem "o prato saudável", isto é, visualizarem que a metade seja preenchida com salada e vegetais, um quarto com amido (batatas ou arroz, por exemplo) e um quarto com uma fonte da proteína (carne, aves, peixe ou soja). 
Além disso, as crianças maiores de dois anos de idade devem realizar pelo menos 30 minutos de atividade física moderada, na maioria dos dias da semana ou, preferencialmente, todos os dias. As crianças podem ser incentivadas a desenvolver mais atividade física no seu estilo de vida, como ir de bicicleta para escola ao invés de ir de carro (se for seguro), subir escadas ao invés de usar elevadores, e ajudar nos afazeres domésticos dentro e fora de casa. Os pais devem ajudar a reduzir o tempo gasto em comportamentos sedentários, como televisão, games, computador ou telefone.

Os profissionais de saúde e os pais devem propor nas suas comunidades que as refeições da escola e os lanches incluam alimentos compatíveis com a saúde cardiovascular, em conjunto com as atividades físicas. Crianças e adolescentes de pais com sobrepeso são de risco para serem ou se tornarem pessoas com sobrepeso, e necessitam de supervisão mais intensa.

Os anos da infância fornecem uma oportunidade original para a promoção da saúde cardiovascular. Os pais procuram ativamente o conselho e o suporte dos médicos para a supervisão da saúde, particularmente quando suas crianças são pequenas. A informação fornecida neste período vulnerável pode ter um impacto importante no estilo de vida futuro. A avaliação e o seguimento de hábitos alimentares, atividade física, além de dados de crescimento, IMC e pressão sangüínea, permitem a identificação dos elementos do estilo de vida que podem contribuir para o risco de obesidade, doenças cardiovasculares e outras co-morbidades mais tarde na vida, 3,6.

A promoção da saúde começa por focalizar a intervenção conforme o nível de desenvolvimento da criança, ao mesmo tempo em que é importante para educar e encorajar os pais, que pessoalmente podem adotar e praticar um estilo de vida voltado à sua própria saúde, especialmente no que se refere à dieta, atividade física e a evitar o uso do tabaco. Desta forma, pais se tornam um modelo positivo para suas crianças e, ao mesmo tempo, melhoram a própria saúde cardiovascular.

Acredita-se que seja esta a forma de prevenção precoce, necessária e adequada, em que os prováveis processos patológicos podem ser atacados na raiz e bloqueados através de orientações que preconizem o estabelecimento de hábitos e rotinas saudáveis, com a perspectiva de estilo de vida saudável e estabelecimento da saúde cardiovascular. É perceptível que a modificação destes resultados possa ser alcançada, melhorando a condição de saúde do indivíduo quando ele ainda é uma criança. Ou seja, promover uma criança sadia é o fundamento para obter um adulto saudável.

Ana Maria de Ulhôa Escobar maria Helena Valente

Referências

I .I Diretriz de Prevenção da Aterosclerose na Infância e Adolescência. Arq Bras Cardiol. 2005;85(Supl 6):3-36.

2.Aguirre P. Socioanthropological aspects of obesity in poverty. In: PAHO Pan American Health Organization. Obesity and poverty: a new public health challenge. Washington (DC): PAHO; 2000. p.II-22.

3.Baker S, Barlow S, Cochran W, Fuchs G, Klish W, Krebs N, et al. Overweight children and adolescents: a clinical report of the North American Society for Pediatric Gastroenterology, Hepatology and Nutrition. J Pediatr Gastroenterol Nutr. 2005;40:533-43.

4.Berenson GS, Srinivasan SR, Bao W. Association between multiple cardiovascular risk factors and atherosclerosis in children and young adults: the Bogalusa Heart Study. N Engl J Med. 1998;338:1650-6.

5.Dietz WH, Robinson TN. Clinical practice. Overweight children and adolescents. N Engl J Med. 2005;352:2100.

6.Dietz WH, Robinson TN. Use of the body mass index (BMI) as a measure of overweight in children and adolescents. J Pediatr. 1998;132:191.

7.Kavey RW, Daniels SR, Lauer RM, Atkins DL, Hayman LL, Taubert K. American Heart Association Guidelines for Primary Prevention of Atherosclerotic Cardiovascular Disease Beginning in Childhood. Circulation. $2003 ; 107: 1562-6$

8.Williams CL, Hayman LL, Daniels SR, Robinson TN, Steinberger J, Paridon $\mathrm{S}$, et al. Cardiovascular health in childhood. A statement for Health Professionals from the Committee on Atherosclerosis, Hypertension, and Obesity in the Young (AHOY) of the Council on Cardiovascular Disease in the Young, American Heart Association. Circulation. 2002;106:143-60. 9.WHO. The world health report 2003: Shaping the future. Neglected global epidemics: three growing threats. Geneva: World Health Organization; 2003.

10. Barreto ACNG, Brasil LMP, Maranhão HS. Sobrepeso: uma nova realidade no estado nutricional de pré-escolares de Natal, RN. Rev Assoc Med Bras. 2007; 53(4):311-6. 